\title{
Mathematical Modeling Describing the Effect of Fishing and Dispersion on Hermaphrodite Population Dynamics
}

\author{
S. Ben Miled ${ }^{1,2} *$, A. Kebir ${ }^{2,3}$ and M. L. Hbid ${ }^{3}$ \\ ${ }^{1}$ ENIT-LAMSIN, University of Tunis el Manar, BP 37, 1002 Tunis Belvédère, Tunisia \\ ${ }^{2}$ Institut Pasteur de Tunis, B.P. 74, 1002 Tunis Belvédère, Tunisia \\ ${ }^{3}$ LMDP, Cadi Ayyad University, BP 2390 Marrakech 4000, Morocco
}

\begin{abstract}
In order to study the impact of fishing on a grouper population, we propose in this paper to model the dynamics of a grouper population in a fishing territory by using structured models. For that purpose, we have integrated the natural population growth, the fishing, the competition for shelter and the dispersion. The dispersion was considered as a consequence of the competition. First we prove, that the grouper stocks may be less sensitive to the removal of large male individuals if female population are totally protected. Second, we show that fishing does not disturb the demographic structure of the population. Finally, we prove that female selective fisheries have the potential of drastically reduce reproductive rates. We also prove that male fishing decreases competition and then increases the total population number.
\end{abstract}

Key words: grouper, fishing, competition for shelters, sex-structured population dynamics, density dependence

AMS subject classification: 39A23, 39A30, 92D40

\section{Introduction}

The impact of fishing on marine populations dynamic is currently approximated by means of theoretical fisheries models. The dynamic of these populations is sustained by the reproduction and is compromised by the fishing activities and the natural mortality caused by diseases, aging, predation or changes in the environmental factors. Models based on mono-specific analysis have been

${ }^{*}$ Corresponding author. E-mail: slimane.benmiled@pasteur.rns.tn 
used to approximate this dynamics. Classical models take into account the fish biology and the measure of its Catch Per Unit of Effort (CPUE) [17].

The goal of this paper is to qualitatively study the effect of fishing effort on hermaphrodite fish populations, taking into account shelter limitation and sexual inversion. This model is applied to the Epinephelinae family of fish like the Grouper; this family is a subfamily of the Serranidae family in the Perciformes order.

Groupers occupy an important position in fish lineages because of the size of their population, the large number of different species and their geographical distribution. For instance, the dusky grouper, Epinephelus marginatus, is found in most seas, except the Black Sea $[14,18]$; the highest population densities are found along the African coasts from Tunisia to Senegal [11]; in the Mediterranean Sea, it lives in sympathy with seven other grouper species [6, 13]; and it has been included in the Red List of The World Conservation Union (IUCN) as one of endangered species (www. iucn.org).

Groupers are protogynous hermaphrodites and are therefore able to change sex. Sex reversal seems to occur when individuals are $9-16$ years old and about $60-90 \mathrm{~cm}$ long (total length) $[5,4,8]$. Large individuals are males and can measure up to $120 \mathrm{~cm}$ and can weigh up to $40 \mathrm{~kg}$. The smaller individual are females and their initial sexual maturity is reached when they are 5 years old and 40 to $50 \mathrm{~cm}$ long [8]. The longevity of these species is long (50 years for Miraglia) and their growth rate is low.

We propose in this paper, a model of the dynamics of this population in a fishing territory, in order to study the impact of fishing on this population. We integrate natural population growth, fishing, competition for shelter and dispersion. Dispersion is considered a consequence of competition. We showed that the grouper population is vulnerable to fishing because the fishing of female individuals has the potential of drastically reducing reproductive rates and sex ratio.

Currently, both structured and global models are used in fishery models. We propose here to use structured models. We subdivide the grouper population in four classes (young, immature adult, female and male) corresponding to four grouper sizes. Age and size are deeply dependent in a grouper population. The obtained model is a system of four difference equations whose analysis encounters mathematical difficulties related to the nature of the non linearity. The stability of the equilibrium and the asymptotic behavior of the solution are discussed in this work.

In the first section, we give the biological precondition necessary to the comprehension of the grouper population dynamics. In the second section, we transform the preceding biological model into a mathematical model. For that, we introduce a structured model in discrete time and give some mathematical elements necessary to its comprehension. In the third section, we study the model through analysis of the equilibrium point. Numerical analysis will be made in order to study the robustness of the model and the impact of fishing on the population. 


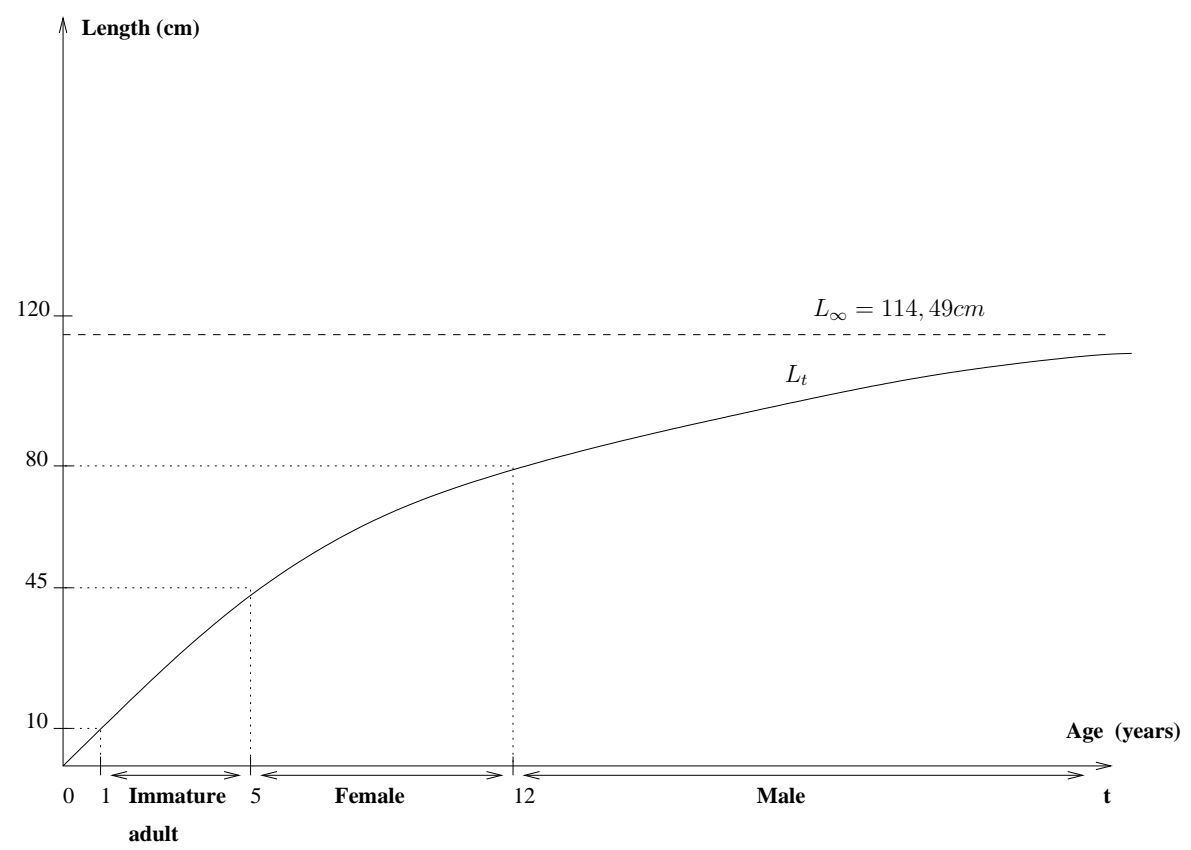

Figure 1: Length/Age relation of Epinephelus marginatus according to the model of Von Bertalanffy: $L_{t}=L_{\infty}\left(1-e^{-k\left(t-t_{0}\right)}\right)$ where $k=0.093$ is the annual growth rate, $t_{0}=-0.75$ is the initial time and $L_{\infty}=114.49$ is the maximum observed size equivalent to the maximum lifespan.

\section{Biological description of the grouper life cycle:}

\subsection{Demographic process}

The grouper is an individual able to produce successively, throughout its life, first female and then male gametes. Therefore, it is suitable to subdivide the population into 4 classes according to the length of the individual. These classes differ by their demographical and ecological parameters.

1. Eggs + Larva: young grouper;

2. Immature adult or juvenile;

3. Female;

4. Male.

Each class has its own duration. Indeed, according to the Length/Age relation for Epinephelus marginatus [8] (figure 1), the time needed to move from the immature adult class to the female class is 4 years.

By spawning, each female releases hundred of eggs, most of them are immediately followed by intense predation by smaller fishes and few of them are fertilized by males. The grouper does 
not make any effort to protect their eggs [20]. After fecundation, the fertilized egg remains in the plankton until it hatches to let the larva out. Next, each larva goes down to the bottom of the sea and occupies a small hole, its shelter. In the spring, when the water warms up, the larva can reach $10 \mathrm{~cm}$ in length and thus passes from the larva class to the immature adult class [6].

The mechanism of sexual inversion is still not entirely understood. We know that it occurs between the ages of 9 and 16 [7] and that size at sexual inversion appears to be an evolution parameter [19]. Some females can reach 26 years old without changing sex [5, 20, 21]. However, a dependence on the sex ratio of the population and social control of the sexual inversion exists [16]. In our case, we assume that sexual inversion occurs for all groupers at a fixed length.

The eggs, whether fertilized or not, as well as the larvae, are submitted to a strong predation pressure, leading to a high death rate [20]. On the other hand, the natural mortality rate is almost zero for the adults (females and males). Anyway the rate of annual survival for this type of fish is generally assumed equal to 0.9 [6]. Indeed, because of their size and diet, adult groupers have very few predators.

\subsection{Fishing process}

The groupers are subject to two types of fishing depending on the size of the individuals: traditional fishing and sport-fishing. Traditional fishing uses hand-lines and affects primarily small females. Sport fishing affects only adult individuals, mainly the large dominant males or the large females.

\subsection{Dispersion process}

There are two types of dispersion: A dispersion which affects the larvae, due to the physical and chemical environmental conditions (water currents, wind, nature of water). The dispersion of the immature adult are due to the competition for shelter. The outcome of the shelter competition between two groups is always in favor of the older group and leads immature adults to completely leave the area [6]. Moreover, the mature adults are sedentary individuals that remain attached to their territory for many years.

\section{Mathematical model:}

\subsection{Parameters definitions}

In order to obtain the general mathematical model that takes into account the preceding assumptions, we subdivided our model into three sub-models: the demographic model, the fishing model and the dispersion model.

In addition, two assumptions were considered in our model:

H1 : The three processes taken into account are: demography, fishing and dispersion.

$\mathrm{H} 2$ : The only limiting factors are the influence of the number of males on the reproduction and the number of surviving adults on the competition for shelters. 
As it was discussed in subsection 2.1., it is suitable to subdivide the population into 4 classes according to body size (young, immature adult, female and male). Let $n_{i}(t)$ be the number of individuals in class $i \in\{1,2,3,4\}$ at time $t$ (by year) and $N_{t}=\left(n_{1}(t), n_{2}(t), n_{3}(t), n_{4}(t)\right)$ be the vector which describes the number of individuals in all classes at time $t$.

We assume that the number of newborn or young depends mostly on the number of females in the territory and their fertility rate, $f$. However, when the number of males, $n_{4}$, is close to 0 , it reduces the fertility rate. Let $g$ be the function which describes the influence of the number of males on the fertility rate. We define $g$ as a positive and differentiable function with a "not deleterious' non linearity (strict depensation or Allee effect) [1, 10]:

$$
g: x \in \mathbb{R}^{+} \longmapsto \frac{C x}{1+C x} \in[0,1], \text { with } C \text { a positive constant. }
$$

The natural mortality rate in a class and the transition rate from one class to the following (by unit of time) depends on the survival and growth conditions. According to assumptions (H2), the survival and the growth conditions are assumed constant. Let $t_{i, i}$ be the fraction of individuals of size class $i \in\{1,2,3,4\}$ who survive and remain in class $i$ after one time unit, and $t_{i+1, i}$ be the fraction of individuals who survive and move to the next larger size category $i+1$. Therefore, if we denote the mortality rate by $m_{i}$ we have, for all $i \in\{1,2,3\}, m_{i}=1-t_{i, i}-t_{i+1, i} \in[0,1]$ and $m_{4}=1-t_{4,4} \in[0,1]$.

For the fishing process, let $\left(p_{i}\right)_{i=1,2,3,4}$ denote the rate of surviving groupers after fishing for classes $i \in\{1,2,3,4\}$, respectively. Therefore, for each $i \in\{1,2,3,4\}, 1-p_{i}$ evaluates the fishing pressures on population class $i$, i.e. it is the probability that an individual of class $i$ is fished by a unit of time, such that for all $i \in\{1,2,3,4\}, p_{i} \in[0,1]$.

In order to define the dispersion model, we define for all $i \in\{1,2,3,4\}, k_{i}$ as the probability of individual of class $i$ staying in the region (i.e. to not dispersing) by unit of time. Therefore, the dispersion rate for each class is equal to $1-k_{i}$. As we note in section 2., the dispersion of the young groupers depends only on the abiotical environment (wind, water currents, nature of water). Hence, $1-k_{1}$, will be taken to be a constant in $[0,1]$. On the other hand, the dispersion process for immature adults depends on the competition for shelter (Hypothesis H2). We assume that this competition is always in favor of the older groupers. According to that we assume that the number of immature adults remaining in the territory after dispersion is proportional to the number of free shelters before dispersion. Therefore, due to the competition for shelter, the dispersion rate is equal to the rate of variation of occupied shelters after natural mortality and fishing. In this case, we assume that if, at time $t, n_{2}(t)+n_{3}(t)+n_{4}(t)<T$ then $k_{2}$ satisfies:

$$
k_{2}\left(n_{2}(t), n_{3}(t), n_{4}(t)\right)=\frac{T-t_{3,2} p_{3} n_{2}(t)-\left(t_{3,3} p_{3}+t_{4,3} p_{4}\right) n_{3}(t)-t_{4,4} p_{4} n_{4}(t)}{T} \in[0,1]
$$

with $T$ the number of shelters in the territory. Otherwise, there is no other juvenile that stays in the site after migration. 
For that, let's define :

$$
k_{2}:(x, y, z) \in \mathbb{R}_{+}^{3} \longmapsto \begin{cases}\frac{T-t_{3,2} p_{3} x-\left(t_{3,3} p_{3}+t_{4,3} p_{4}\right) y-t_{4,4} p_{4} z}{T} & \text { if } x+y+z \leq T, \\ 0 & \text { otherwise }\end{cases}
$$

Anyway, if initial population condition satisfies $n_{1}(0), n_{2}(0), n_{3}(0)$ and $n_{4}(0)<T$ and model parameters satisfy $f<\frac{1}{k_{1} p_{1}}$ and $\left(t_{3,2}+t_{3,3}\right) p_{3}+\left(t_{4,3}+t_{4,4}\right) p_{4}<1$ then for each $t \geq 0$ $k_{2}\left(n_{2}(t), n_{3}(t), n_{4}(t)\right)$ is in $[0,1]$.

\subsection{Model equations}

Considering all the elements defined so far we get the following non linear equation:

$$
N_{t+1}=M\left(n_{2}(t), n_{3}(t), n_{4}(t)\right) P L\left(n_{4}(t)\right) N_{t},
$$

with $M\left(n_{2}, n_{3}, n_{4}\right)$ : the density dependent matrix associated with the dispersion process, $P$ : the fishing matrix and $L\left(n_{4}\right)$ : the density dependent matrix associated with the demographic process.

These matrices are defined as follows:

$$
M\left(n_{2}, n_{3}, n_{4}\right)=\left(\begin{array}{cccc}
k_{1} & 0 & 0 & 0 \\
0 & k_{2}\left(n_{2}, n_{3}, n_{4}\right) & 0 & 0 \\
0 & 0 & k_{3} & 0 \\
0 & 0 & 0 & k_{4}
\end{array}\right), P=\left(\begin{array}{cccc}
p_{1} & 0 & 0 & 0 \\
0 & p_{2} & 0 & 0 \\
0 & 0 & p_{3} & 0 \\
0 & 0 & 0 & p_{4}
\end{array}\right)
$$

and

$$
L\left(n_{4}\right)=\left(\begin{array}{cccc}
t_{1,1} & 0 & f g\left(n_{4}\right) & 0 \\
t_{2,1} & t_{22} & 0 & 0 \\
0 & t_{3,2} & t_{3,3} & 0 \\
0 & 0 & t_{4,3} & t_{4,4}
\end{array}\right)
$$

Therefore, the final model corresponds to the following non-linear system of difference equations:

$$
\left\{\begin{array}{l}
n_{1}(t+1)=k_{1} p_{1} t_{1,1} n_{1}(t)+k_{1} p_{1} f g\left(n_{4}(t)\right) n_{3}(t) \\
n_{2}(t+1)=k_{2}\left(n_{2}(t), n_{3}(t), n_{4}(t)\right) p_{2} t_{2,1} n_{1}(t)+k_{2}\left(n_{2}(t), n_{3}(t), n_{4}(t)\right) p_{2} t_{2,2} n_{2}(t) \\
n_{3}(t+1)=k_{3} p_{3} t_{3,2} n_{2}(t)+k_{3} p_{3} t_{3,3} n_{3}(t) \\
n_{4}(t+1)=k_{4} p_{4} t_{4,3} n_{3}(t)+k_{4} p_{4} t_{4,4} n_{4}(t)
\end{array}\right.
$$

\section{Model Analysis}

From the following equilibrium equations, 


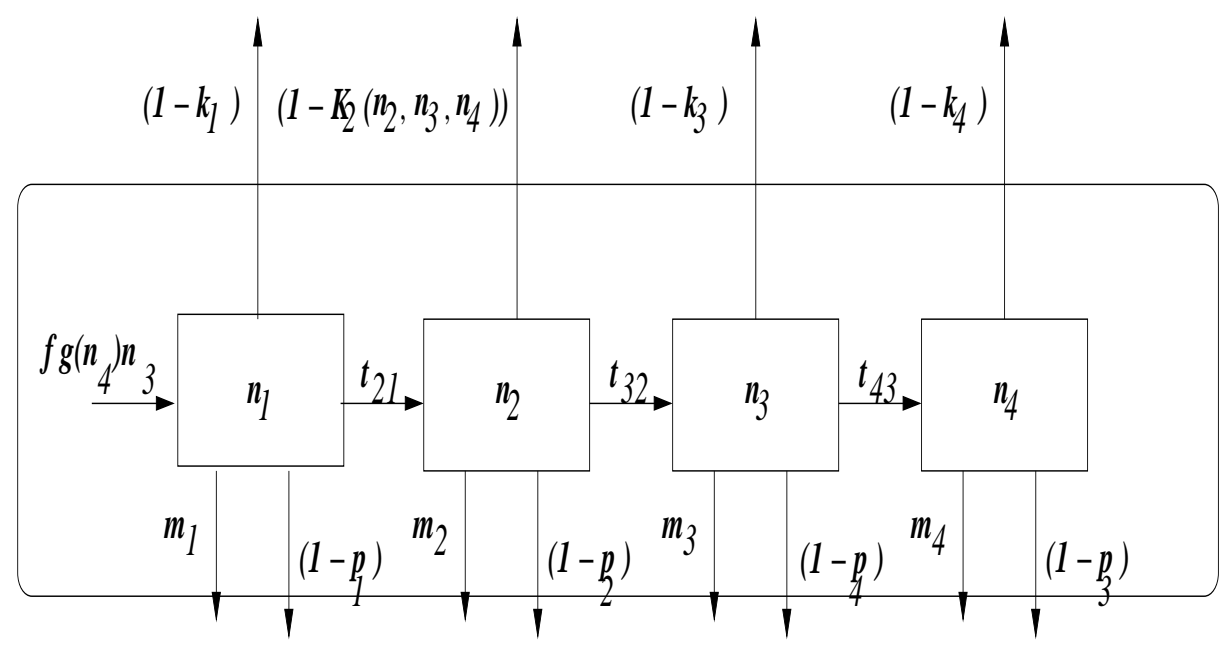

Figure 2: Mathematical Model for Grouper Population.

$$
\left\{\begin{array}{l}
n_{1}=A_{1} g\left(n_{4}\right) n_{3}+B_{1} n_{1} \\
n_{2}=A_{2}\left(n_{2}, n_{3}, n_{4}\right) n_{1}+B_{2}\left(n_{2}, n_{3}, n_{4}\right) n_{2} \\
n_{3}=A_{3} n_{2}+B_{3} n_{3} \\
n_{4}=A_{4} n_{3}+B_{4} n_{4}
\end{array}\right.
$$

with $A_{1}=k_{1} p_{1} f, A_{i}=k_{i} p_{i} t_{i, i-1}(i=2,3,4)$ and $B_{i}=k_{i} p_{i} t_{i, i}(i=1,2,3,4)$.

It is clear that $\mathbf{0}=(0,0,0,0)$ is a solution of (4.1), which corresponds to the extinction equilibrium state. Moreover, equations (4.1) is equivalent to:

$$
\left\{\begin{array}{l}
n_{1}=\alpha_{1} \alpha_{3} g\left(\alpha_{4} n_{2}\right) n_{2} \\
n_{2}=\alpha_{1} \alpha_{3} \frac{A_{2}\left(n_{2}, \alpha_{3} n_{2}, \alpha_{4} n_{2}\right)}{1-B_{2}\left(n_{2}, \alpha_{3} n_{2}, \alpha_{4} n_{2}\right)} g\left(\alpha_{4} n_{2}\right) n_{2} \\
n_{3}=\alpha_{3} n_{2} \\
n_{4}=\alpha_{4} n_{2}
\end{array}\right.
$$

where, $\forall j \in\{3,4\}, \alpha_{j}=\prod_{k=3}^{j} \frac{A_{k}}{1-B_{k}}$ and $\alpha_{1}=\frac{A_{1}}{1-B_{1}}$ such that for all $i \in\{1,2,3,4\},(1-$ $\left.B_{i}\right) \neq 0$.

From (4.2) it is obvious that only the trivial equilibrium has $n_{2}=0$ and if $n_{2}>0$ then the non trivial equilibrium point has to satisfy:

$$
1=\alpha_{1} \alpha_{3} \frac{A_{2}\left(n_{2}, \alpha_{3} n_{2}, \alpha_{4} n_{2}\right)}{1-B_{2}\left(n_{2}, \alpha_{3} n_{2}, \alpha_{4} n_{2}\right)} g\left(\alpha_{4} n_{2}\right)
$$


where at equilibrium, the juvenile migration function satisfies:

$$
k_{2}\left(n_{2}, n_{3}, n_{4}\right)=1-\alpha_{2} n_{2}
$$

with, $\alpha_{2}=\frac{1}{T}\left(t_{3,2} p_{3}+\left(t_{3,3} p_{3}+t_{4,3} p_{4}\right) \alpha_{3}+t_{4,4} p_{4} \alpha_{4}\right)$ and then,

$$
A_{2}\left(n_{2}, \alpha_{3} n_{2}, \alpha_{4} n_{2}\right)=p_{2} t_{2,1}\left(1-\alpha_{2} n_{2}\right) \text { and } B_{2}\left(n_{2}, \alpha_{3} n_{2}, \alpha_{4} n_{2}\right)=p_{2} t_{2,2}\left(1-\alpha_{2} n_{2}\right)
$$

Since the equation (4.3) is equivalent to the following second degree equation:

$$
C \alpha_{2} \alpha_{4} p_{2}\left(t_{2,2}+\alpha_{1} \alpha_{3} t_{2,1}\right) n_{2}^{2}+C \alpha_{4}\left(1-p_{2}\left(t_{2,2}+\alpha_{1} \alpha_{3} t_{2,1}\right)\right)+p_{2} t_{2,2} \alpha_{2} n_{2}+=1-p_{2} t_{2,2} 0
$$

then, let's $a=C \alpha_{2} \alpha_{4} p_{2}\left(t_{2,2}+\alpha_{1} \alpha_{3} t_{2,1}\right), b=C \alpha_{4}\left(1-p_{2}\left(t_{2,2}+\alpha_{1} \alpha_{3} t_{2,1}\right)\right)+p_{2} t_{2,2} \alpha_{2}$ and $c=1-p_{2} t_{2,2}$.

The next proposition expresses a necessary and sufficient condition for system (3.2) to possess positive equilibrium points.

Proposition 1. System (3.2) has positive equilibrium points if and only if $b \leq-2 \sqrt{a c}$ (see figure $3)$.

Moreover,

- if $b=-2 \sqrt{a c}$ then the positive equilibrium point is unique and is given as follows:

$$
N^{*}=\left(n_{1}^{*}, n_{2}^{*}, n_{3}^{*}, n_{4}^{*}\right)=\frac{-b}{2 a}\left(\alpha_{1} \alpha_{3} g\left(-\alpha_{4} \frac{b}{2 a}\right), 1, \alpha_{3}, \alpha_{4}\right)
$$

- if $b<-2 \sqrt{a c}$ then there exist two positive equilibrium points, given by:

1.

$$
N_{1}^{*}=\left(n_{1_{1}}^{*}, n_{2_{1}}^{*}, n_{3_{1}}^{*}, n_{4_{1}}^{*}\right)=\frac{-b-\sqrt{b^{2}-4 a c}}{2 a}\left(\alpha_{1} \alpha_{3} g\left(-\alpha_{4} \frac{b+\sqrt{b^{2}-4 a c}}{2 a}\right), 1, \alpha_{3}, \alpha_{4}\right)
$$

2.

$$
N_{2}^{*}=\left(n_{1_{2}}^{*}, n_{2_{2}}^{*}, n_{3_{2}}^{*}, n_{4_{2}}^{*}\right)=\frac{-b+\sqrt{b^{2}-4 a c}}{2 a}\left(\alpha_{1} \alpha_{3} g\left(-\alpha_{4} \frac{b-\sqrt{b^{2}-4 a c}}{2 a}\right), 1, \alpha_{3}, \alpha_{4}\right)
$$

Proof. As equation (4.3) is equivalent to a second degree equation, it is easy to show the existence of the equilibrium points when $b^{2}-4 a c \geq 0$ and by a simple calculation their formulas (see figure $3)$.

On the other hand, since $a$ and $c$ are positive quantities, positivity of these equilibrium points needs the condition $b<0$.

Indeed, if $b<0$ we have: 


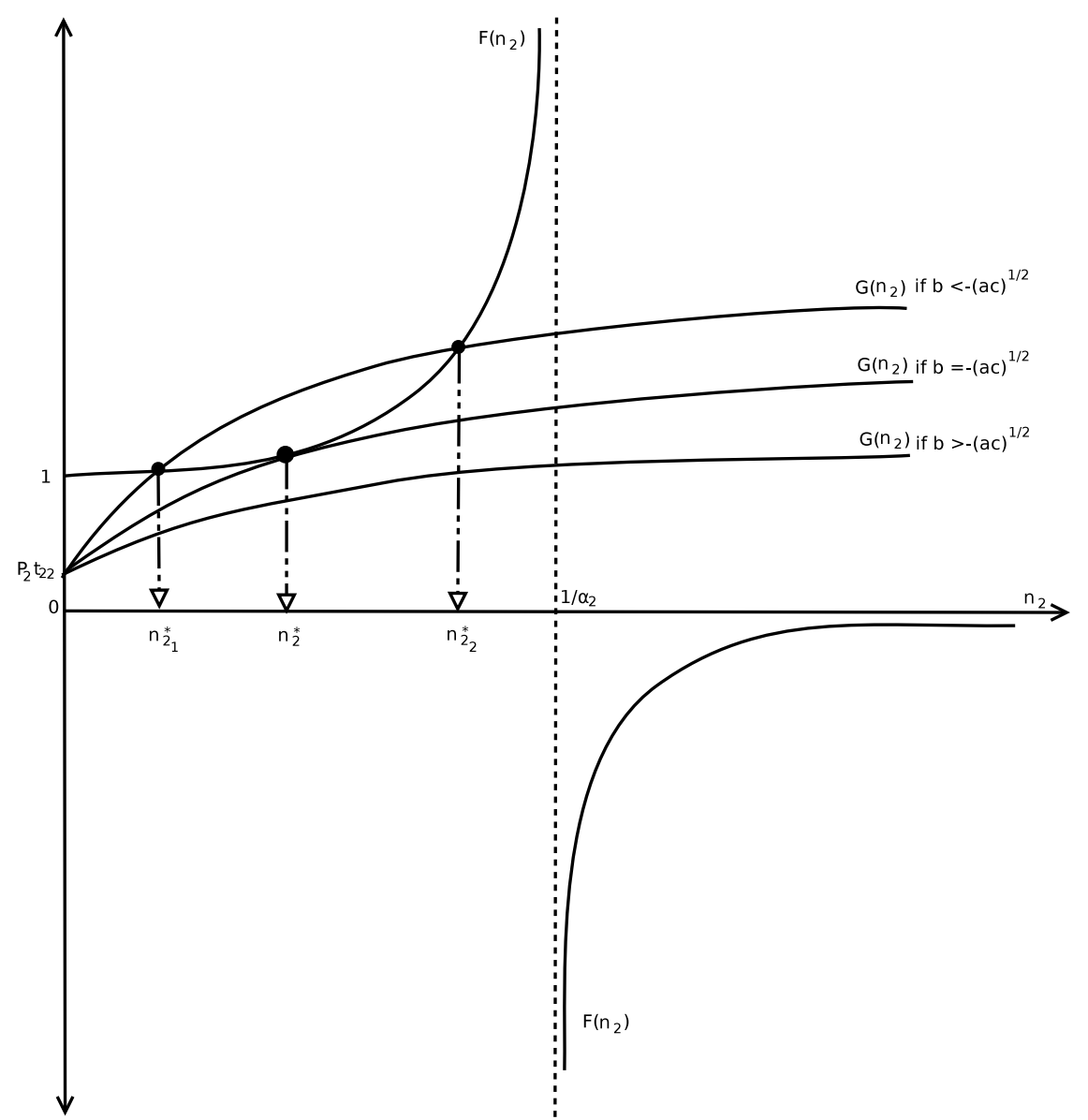

Figure 3: Intersection points between the curves of the functions $F(x)=\frac{1}{1-\alpha_{2} x}$ and $G(x)=$ $p_{2}\left(t_{2,1} \alpha_{1} \alpha_{3} \frac{C \alpha_{4} x}{1+C \alpha_{4} x}+t_{2,2}\right)$ for $\left.x \in\right] 0,1 / \alpha_{2}$ [ for different values of $b$ in relation to $a$ and $c$, where $a=C \alpha_{2} \alpha_{4} p_{2}\left(t_{2,2}+\alpha_{1} \alpha_{3} t_{2,1}\right), b=C \alpha_{4}\left(1-p_{2}\left(t_{2,2}+\alpha_{1} \alpha_{3} t_{2,1}\right)\right)+p_{2} t_{2,2} \alpha_{2}$ and $c=1-p_{2} t_{2,2}$.

$$
\sqrt{b^{2}-4 a c} \leq-b \text { and } 0<\frac{-b}{2 a}<\frac{1}{2 \alpha_{2}}
$$

and then,

$$
0<\frac{-b-\sqrt{b^{2}-4 a c}}{2 a} \leq \frac{-b}{2 a} \leq \frac{-b+\sqrt{b^{2}-4 a c}}{2 a}<\frac{1}{\alpha_{2}}
$$

which proves the result in (1).

A particular case of the model (3.1) is when the number of shelter $T$ tends to infinity i.e. where there is no refuge limitation and then $k_{2}$ is equal to 1 . In this case, system (3.2) admits a unique positive equilibrium point if and only if $b<0$, where $\alpha_{2}=0$. Moreover, the equilibrium point reads as follows: 


$$
N_{3}^{*}=\left(n_{1_{3}}^{*}, n_{2_{3}}^{*}, n_{3_{3}}^{*}, n_{4_{3}}^{*}\right)=\frac{-b}{c}\left(\alpha_{1} \alpha_{3} g\left(-\alpha_{4} \frac{b}{c}\right), 1, \alpha_{3}, \alpha_{4}\right)
$$

For this particular case with no refuge limitation i.e. $T \rightarrow+\infty$ we can only assure that the Jacobian matrix of system (3.2) when $k_{2}=1$ is non-negative, irreducible and primitive. Then by Perron-Frobenius theorem there is a positive strictly dominant simple eigenvalue $r>0$ depending on model parameter. If $r<1$ then the equilibrium $N_{3}^{*}$ is locally asymptotically stable.

Another particular case is when $k_{2}\left(n_{2}(t), n_{3}(t), n_{4}(t)\right)=0, \forall t \geq 0$, i.e. at each time $t \geq 0$ all juveniles leave the territory and then $n_{2}(t)=0, \forall t \geq 0$. Therefore, the system (3.2) does not admit positive equilibrium points and becomes:

$$
\left\{\begin{array}{l}
n_{1}(t+1)=B_{1} n_{1}(t)+A_{1} g\left(n_{4}(t)\right) n_{3}(t) \leq B_{1} n_{1}(t)+A_{1} n_{3}(t) \\
n_{3}(t+1)=B_{3} n_{3}(t) \\
n_{4}(t+1)=A_{4} n_{3}(t)+B_{4} n_{4}(t)
\end{array}\right.
$$

If we consider now the system (4.4), we note that $B_{1}, B_{3}$ and $B_{4} \in(0,1]$ implies that the strictly dominant eigenvalue of the associated matrix is less than 1 and so for any initial condition $\left(n_{1}(0), n_{3}(0), n_{4}(0)\right)$ the corresponding solution verifies $\lim _{t \rightarrow+\infty}\left(n_{1}(t), n_{3}(t), n_{4}(t)\right)=(0,0,0)$. This result induces that if $k_{2}\left(n_{2}(t), n_{3}(t), n_{4}(t)\right)=0, \forall t \geq 0$, then equilibrium 0 of system (3.2) is globally asymptotically stable.

Next, we are interested in the asymptotic behavior of system (3.2) out of these particular cases.

The trivial, or extinction, equilibrium $\mathbf{0}=(0,0,0,0)$ of system (3.2) always exists. The Jacobian of system (3.2) at $\mathbf{0}$ is:

$$
M(0) P L(0)=\left(\begin{array}{cccc}
B_{1} & 0 & 0 & 0 \\
p_{2} t_{2,1} & p_{2} t_{2,2} & 0 & 0 \\
0 & A_{3} & B_{3} & 0 \\
0 & 0 & A_{4} & B_{4}
\end{array}\right)
$$

and thus the dominant eigenvalue is always less than 1 since we have, for all $i \in\{1,3,4\}$, $0<B_{i}<1$ and $0<p_{2} t_{2,2}<1$, which yields the following result:

Proposition 2. The trivial equilibrium $\mathbf{0}=(0,0,0,0)$ of system (3.2) is always locally asymptotically stable.

This result is a common feature of sexually-reproducing populations i.e. an initially low population density produces the extinction of the population, displaying the Allee effect [1].

The stability analysis of the positive equilibrium point is difficult to analyze due to the huge number of parameters. Therefore, in the next section we are presenting a set of simulations which completes the study of those cases where the performed analysis does not help in characterizing the asymptotic behavior of solutions. These unknown cases correspond to the situation of existence of a positive equilibrium point for which it is not known whether it is stable or not. 


\section{Numerical Results:}

In this section we pursue the analysis of the stability and the asymptotic behavior of solutions of system (3.2) by means of numerical simulations.

Firstly, we start by fixing the values of the parameters from the literature. For missing parameter values, we make a parameter estimation by means of the stable population with an expected maximal exploitation rate in a territory without fishing. We do that in order to study the fishing effect on the population on optimal condition environment. Secondly, we make a study of the model's robustness for these parameters. Finally, we numerically analyze the effect of the fishing on a stable grouper population with favorable survival conditions.

\subsection{Parameters values:}

The mature adults are sedentary thus we assume that $k_{3}=k_{4}=1$. On the other hand, as noted in sections (2.) and (3.), the dispersion rate of immature adult, $1-k_{2}$, depends on the number of shelters in the territory. In this numerical study, we consider an arbitrary number of shelters, $T$, equal to 1000 .

Generally for fish, the fertility rate (i.e. potentially fertilized eggs number per matting) is hard to calculate. Indeed, this rate depends not only on female size, but also on ecological and physicchemical parameters that are difficult to be measured. Therefore, the fertility rate, $f$, is assumed to be 2 , this corresponds to a minimal state of balance in a sexual reproduction.

We assume that the mortality rate of each class is equal to the average natural mortality rate of the entire grouper population. According to biological data [6], $\left(m_{i}\right)_{i \leq 4}$ are equal to 0.10. Therefore, using 3.1., $t_{i, i}+t_{i+1, i}=1-m_{i}=0.9$ and $t_{4,4}=0.9$. Moreover, as the duration of the young class is equal to 1 year, the time unit of our model, then $t_{1,1}=0$ and $t_{2,1}=0.9$.

Next, let us define $\tau_{i}$ be the limiting duration per year for each class $i \in\{1,2,3,4\}$. Indeed, beside the hypothesis of uniform average mortality rate, we also assume a uniform age distribution of the population number in each class so the rate of individuals of the class $i$ that can pass to the class $i+1$ is less than $\frac{1}{\tau_{i}}$, for each $i \in\{2,3\}$. According to the Length/Age relation (see figure 1 ), we have $\tau_{1}=1, \tau_{2}=4, \tau_{3}=7$ and if the maximum lifespan for grouper is 50 years, we have $\tau_{4}=38$. Consequently, $t_{3,2} \leq \frac{1}{4}$ and $t_{4,3} \leq \frac{1}{7}$.

Moreover, we assume a uniform size distribution of the individuals inside each class. Thus, if $N^{*}=\left(n_{i}{ }^{*}\right)_{i \leq 4}$ is a positive equilibrium point, the size distribution of the population at the equilibrium point $N^{*}$, is

We are now able to fix the remaining parameters, $\left(k_{1}, t_{3,2}, t_{4,3}\right)$. For that, we will fix our parameters such that the expected exploitation rate $E$ is equal to the maximum exploitation rate, $E_{\max }$ when the population is at a stable equilibrium state without fishing. Let us recall that the exploitation rate corresponds to the ratio of natural mortality by all causes of disappearance from the territory [15]. That coincides with an optimal territory in term of fisheries management.

Therefore, we look first for the stable equilibrium states without fishing. In this case, the complete model is defined as follows, 


\begin{tabular}{|c|c|c|c|c|}
\hline Young Ind. Number & 1 & 2 & $\ldots$ & $n_{1}^{*}$ \\
\hline Size & $L(0)$ & $L\left(\frac{\tau_{1}}{n_{1}^{*}}\right)$ & $\ldots$ & $L\left(\frac{\left(n_{1}^{*}-1\right) \tau_{1}}{n_{1}^{*}}\right)$ \\
\hline \hline Juvenile Ind. Number & 1 & 2 & $\ldots$ & $n_{2}^{*}$ \\
\hline Size & $L\left(\tau_{1}\right)$ & $L\left(\tau_{1}+\frac{\tau_{2}}{n_{2}^{*}}\right)$ & $\ldots$ & $L\left(\tau_{1}+\frac{\left(n_{2}^{*}-1\right) \tau_{2}}{n_{2}^{*}}\right)$ \\
\hline \hline Female Ind. Number & 1 & 2 & $\ldots$ & $n_{3}^{*}$ \\
\hline Size & $L\left(\sum_{i=1}^{2} \tau_{i}\right)$ & $L\left(\sum_{i=1}^{2} \tau_{i}+\frac{\tau_{3}}{n_{3}^{*}}\right)$ & $\ldots$ & $L\left(\sum_{i=1}^{2} \tau_{i}+\frac{\left(n_{3}^{*}-1\right) \tau_{3}}{n_{3}^{*}}\right)$ \\
\hline \hline Male Ind. Number & 1 & 2 & $\ldots$ & $n_{4}^{*}$ \\
\hline Size & $L\left(\sum_{i=1}^{3} \tau_{i}\right)$ & $L\left(\sum_{i=1}^{3} \tau_{i}+\frac{\tau_{4}}{n_{4}^{*}}\right)$ & $\ldots$ & $L\left(\sum_{i=1}^{3} \tau_{i}+\frac{\left(n_{4}^{*}-1\right) \tau_{4}}{n_{4}^{*}}\right)$ \\
\hline
\end{tabular}

Table 1: Population length distribution of the population at the equilibrium point $N^{*}=$ $\left(n_{1}^{*}, n_{2}^{*}, n_{3}^{*}, n_{4}^{*}\right)$, assuming a uniform age distribution and Von Bertalanffy Length/Age relation (figure 1).

$$
N_{t+1}=D N_{t} \text { with } D=M L .
$$

First, we seek the parameter sets in which we have at least an acceptable equilibrium point (i.e. $\left.n_{i}^{*}>0\right)$ of equation (5.1).

By a simple numerical analysis we found that this equation admits two positive equilibrium points $N_{1}^{*}=\left(n_{i_{1}}^{*}\right)_{i \leq 4}$ and $N_{2}^{*}=\left(n_{i_{2}}^{*}\right)_{i \leq 4}$, for each $\left(k_{1}, t_{3,2}, t_{4,3}\right) \in[0,1] \times\left[0, \frac{1}{4}\right] \times\left[0, \frac{1}{7}\right]$. Moreover, for these parameter ranges, the equilibrium solutions satisfy $n_{i_{1}}^{*}\left(k_{1}, t_{3,2}, t_{4,3}\right)<1$ and $n_{i_{2}}^{*}\left(k_{1}, t_{3,2}, t_{4,3}\right)>1$. Therefore, for the maximization of the exploitation rate we choose the second equilibrium point $N_{2}^{*}$.

Next, let the expected exploitation rate, $E$, be the ratio of natural mortality, $m$, compared to $Z$ the number of exploitable individuals at equilibrium (i.e. juvenile, female and male) who left the territory by natural mortality and dispersion i.e.

$$
E=\frac{m}{Z}
$$

According to the work of Beverton and Holt [3], we can approach $Z$ by:

$$
Z=k\left(L_{\infty}-L_{m}\right) /\left(L_{m}-L_{c}\right)
$$

where,

- $L_{\infty}$ is the maximum observed size equivalent to the maximum lifespan and $k$ the mean annual growth rate of the fish specie (see figure 1).

- $L_{m}$ the average length of the population at the equilibrium point,

- $L_{c}$ the median size of the population. 
For the calculation of $L_{m}$ and $L_{c}$, we use the population length distribution of the population at an equilibrium point, see table (5.1.).

Finally, we build a grid on $[0,1] \times\left[0, \frac{1}{4}\right] \times\left[0, \frac{1}{7}\right]\left(\left(k_{1}, t_{3,2}, t_{4,3}\right) \in[0,1] \times\left[0, \frac{1}{4}\right] \times\left[0, \frac{1}{7}\right]\right)$, and analyze the spectrum of the Jacobian at $N_{2}^{*}$ and seek $E_{\max }$ on each vertex of the grid. We prove that the maximum expected exploitation rate for a stable non trivial equilibrium point is obtained for,

$$
\left(t_{1,1}, t_{2,2}, t_{3,3}, t_{4,4}, t_{2,1}, t_{3,2}, t_{4,3}, k_{1}, k_{3}, k_{4}\right)=(0,0.80,0.81,0.9,0.9,0.10,0.09,0.7,1,1)
$$

and we have $E_{\max }=0.19$ and $N_{2}^{*}=(230,314,165,148)$.

\subsection{Sensitivity analysis}

A model is known as insensitive to small variations of parameters for which it was optimized, if this model produces small variations in the result when subject to small modifications of these parameters.

In our case, we will study the sensitivity of the model with respect to parameters $k_{1}, t_{3,2}$ and $t_{4,3}$ in such a way that the exploitation rate remains maximum.

The technique we used consist in fixing at each time one of the three parameters at its optimal value, i.e. for which the exploitation rate, $E$, is maximum and to make the two others vary on a normalized range of variations, $[0,1]$, in order to compare the exploitation rate and the maximum exploitation rate, $E_{\max }$. For that, first, we fix the transition rate, $t_{4,3}$, at its optimal value $t_{4,3}^{*}$. Next, we study the variation of $\left(E_{\max }-E\right)$ for $k_{1} \in\left[k_{1}^{*}-0.05 k_{1}^{*}, k_{1}^{*}+0.05 k_{1}^{*}\right]$ and $t_{3,2} \in$ $\left[t_{3,2}^{*}-0.05 t_{3,2}^{*}, t_{3,2}^{*}+0.05 t_{3,2}^{*}\right]$ where, $k_{1}^{*}$ and $t_{3,2}^{*}$ correspond respectively to the optimal values of the young dispersion rate and the transition rate of the female class to the male class. Second, we fix the young dispersion rate, $k_{1}$, at $k_{1}^{*}$ and move, $t_{3,2}$ in $\left[t_{3,2}^{*}-0.05 t_{3,2}^{*}, t_{3,2}^{*}+0.05 t_{3,2}^{*}\right]$ and $t_{4,3}$ in $\left[t_{4,3}^{*}-0.05 t_{4,3}^{*}, t_{4,3}^{*}+0.05 t_{4,3}^{*}\right]$. Third, we fix $t_{3,2}$, in its optimal value and move $k_{1}$ in $\left[k_{1}^{*}-0.05 k_{1}^{*}, k_{1}^{*}+0.05 k_{1}^{*}\right]$ and $t_{4,3}$ in $\left[t_{4,3}^{*}-0.05 t_{4,3}^{*}, t_{4,3}^{*}+0.05 t_{4,3}^{*}\right]$.

It can be noticed from figures (4(a)) and (4(b)) that for all $k_{1} \in\left[k_{1}^{*}-0.05 k_{1}^{*}, k_{1}^{*}+0.05 k_{1}^{*}\right]$ the curve $E_{\max }-E$ behaves in a single manner so that the exploitation rate is insensitive with respect to parameter $k_{1}$ in the neighborhood of $k_{1}^{*}$. On the other hand, we remark from figures (4(a)) and (4(c)) that the curve shows a sensitivity for the transition rate $t_{3,2}$ on the right side of $t_{3,2}^{*}$ more than on left side. This sensitivity is greater, compared to $t_{4,3}$. Indeed, the growth is linear starting from $t_{3,2}^{*}$ (Figures 4(b) and 4(c)). In all cases, $\left(E_{\max }-E\right) / E_{\max }$ is less than $210^{-2}$.

\subsection{The effect of the fishing parameters:}

In this paragraph, we will study the effect of fishing on a stable population of groupers in favorable survival conditions. The fishing rates $1-p_{i}$ constitute important parameters of the model since they characterize the fishing effort on the population.

As was discussed in subsection 2.2., the fishing affects especially the adult class. So we suppose in the sequel that there is no fishing for immature classes, i.e. $p_{1}=p_{2}=1$, and we perform 


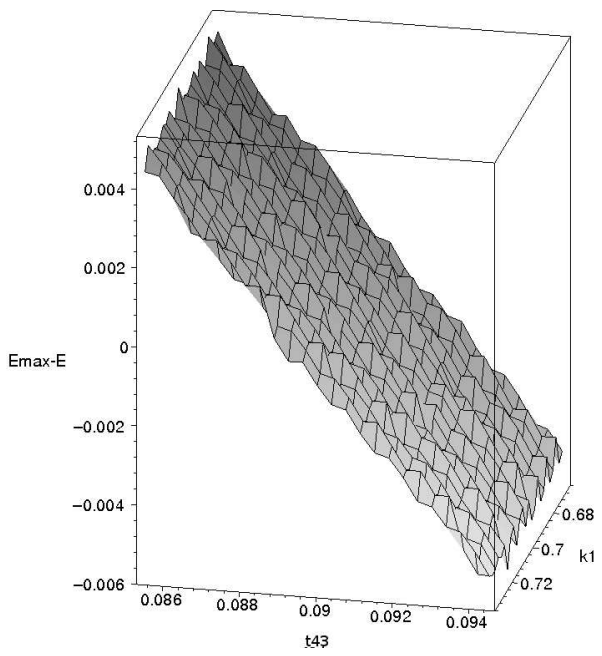

(a) $t_{3,2}=t_{3,2}{ }^{*}$.

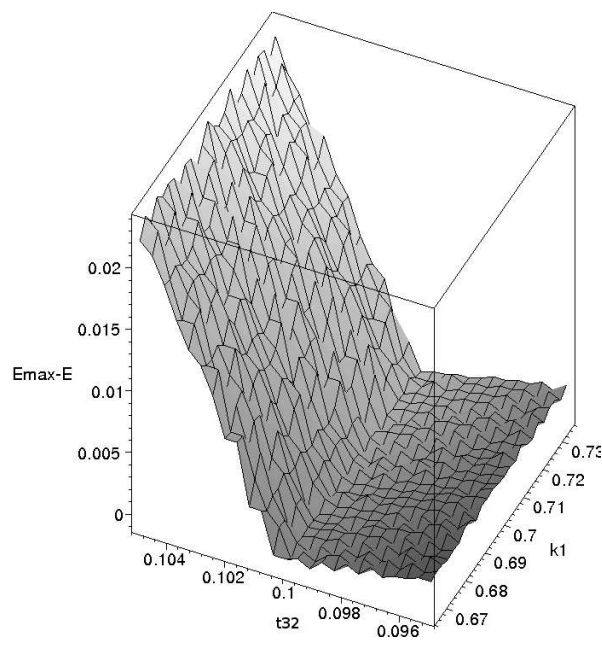

(b) $t_{4,3}=t_{4,3} *$.

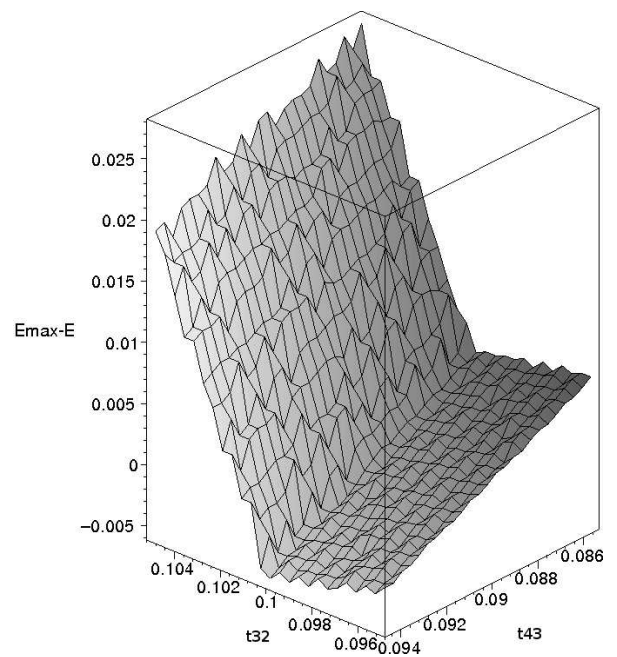

(c) $k_{1}=k_{1}{ }^{*}$.

Figure 4: The variation of $\left(E_{\max }-E\right)$ for $t_{3,2}=t_{3,2}{ }^{*}=0.05, t_{4,3}=t_{4,3}{ }^{*}=0.09$ and $k_{1}=k_{1}{ }^{*}=$ 0.7 . 
numerical simulations on female and male fishing, $p_{3}$ and $p_{4}$, respectively. For that we will perturb the non trivial equilibrium point of the model corresponding to equation (5.1).

In the first plot of Fig 5, we analyze the effect of a higher female fishing effort bigger than 0.7 and for different value of male fishing efforts on the different classes of the population. We can notice from these figures that for all $p_{3} \in[0.7,1]$ and $p_{4} \in\{0.1,0.5,1\}$, the individuals number for each subclass increases with female fishing $p_{3}$ and that the growth coefficient increases with male fishing $p_{4}$.

Now considering a fixed value of $p_{3} \in\{0.8,0.9,1\}$ and for all $p_{4} \in[0.1,1]$ (figure 6), the maximum of the individual number of each class, except the male ones, is reached for $p_{3}=1$ and $p_{4}<1$. We observe the same phenomenon for the total population number at the equilibrium state $N_{2}^{*}, T_{p}$ (figure 7) and for the exploitation rate, $E$ (figure 8). This phenomenon can be explained by the fact that male fishing favors the integration of immature adults in the territory. Moreover, as the male number is smaller than the total number of individual from the other classes, the total population dynamical behavior likes female one. This leads to an asymmetry of the effect of fishing on the total population.

\section{Discussion and conclusion}

In Tunisia, the grouper is subjected to two types of fishing, one known as "traditional", and the other known as "recreational" fishing or "sport-fishing". Traditional fishing uses hand-lines, lines of more than 100 hooks; the fishing effort depends on the number of hooks by boat. This fishing affects primarily the small females. The other type of fishing is practiced either as a sport or by some sponge fishermen as a wage complement. It affects only the large individuals, mainly the large dominant males or the large females. This type of fishing destabilizes the population, especially if the females are fished before reproduction. On the other hand, as we proved in this paper, male fishing favors the integration of the young in the territory, which implies that, without females fishing, the maximum number of individuals in the total population is reached when the male fishing rate, $\left(1-p_{4}\right)$, is equal to 0.5 (figure 6). Consequently, the grouper stocks may be less sensitive to the removal of large individuals (male) if female population are totally protected. This result has already been observed by Alonzo and Mangel [2] for sex-changing fish.

On the other hand, even if there is no fishing pressure on the males, if more than $30 \%$ of the female classes are fished, the grouper population is predicted to die out. This shows that the grouper population may be vulnerable to fishing. Size selective fisheries have the potential of drastically reducing reproductive rates and thus the sex ratio, the size-dependent fecundity and the spawning in aggregation size, as was also observed in [2]. This explains that one of the most important dynamical characteristics of the grouper, the fact that it is a protogynous hermaphrodite.

From the numerical results, we note that fishing does not disturb the demographic structure of the population since the population pyramid at the equilibrium point solution keeps always an expansion aspect, i.e. broad base and narrow top. This is in agreement with the observations made by Chauvet [9] on the demographic structure of the grouper populations present in the southern part of the Western Mediterranean basin and on the pacific ocean. Chauvet showed that, in the 


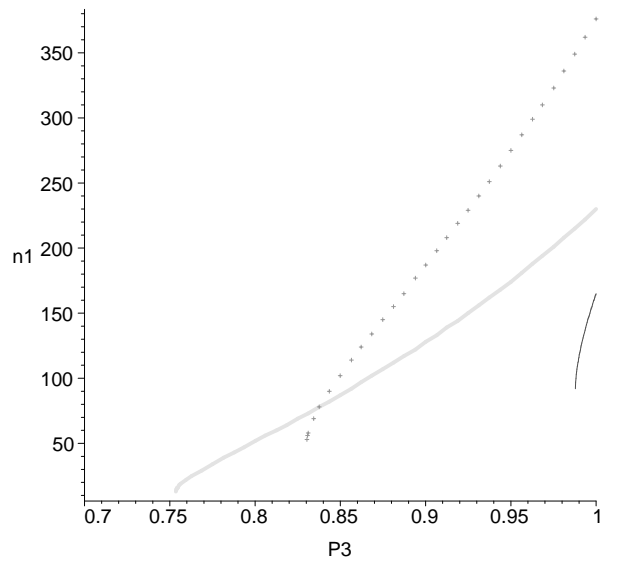

$p 4=0.1$
$p 4=0.5$
$p 4=1$

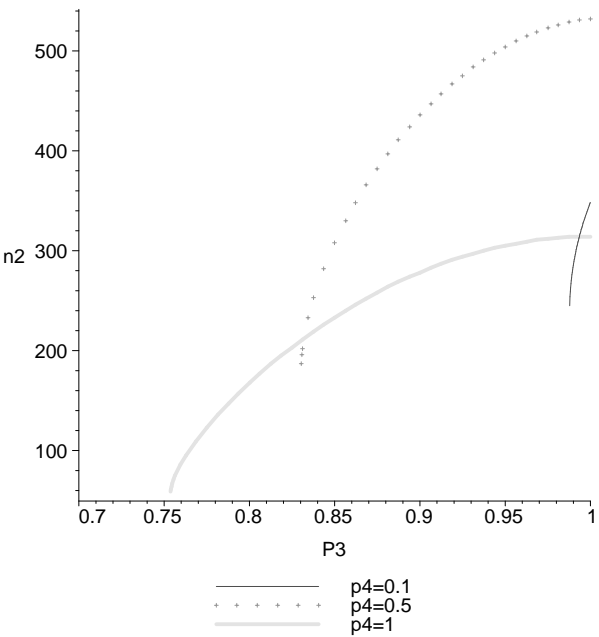

(b) Effect of fishing on the immature adult class

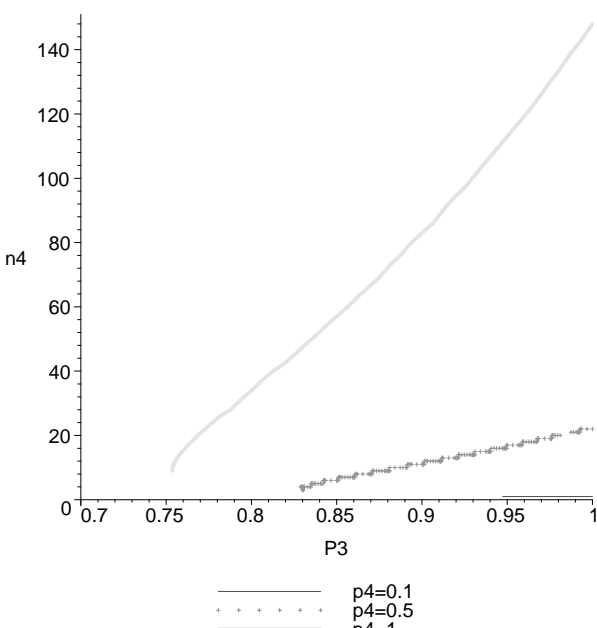

(d) Effect of fishing on the male class

(c) Effect of fishing on the female class

Figure 5: Effect of fishing on the grouper population for $p_{3} \in[0.7,1], p_{4} \in\{0.1,0.5,1\}$. 


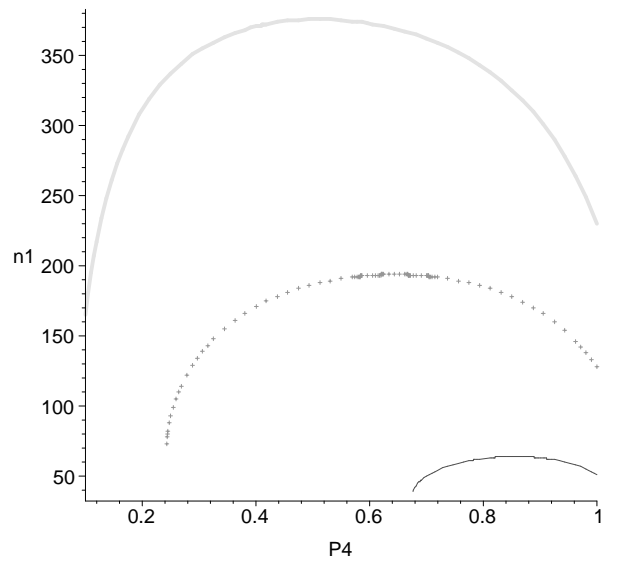

$\mathrm{p} 3=0.8$
$\mathrm{p} 3=0.9$
$\mathrm{p} 3=1$

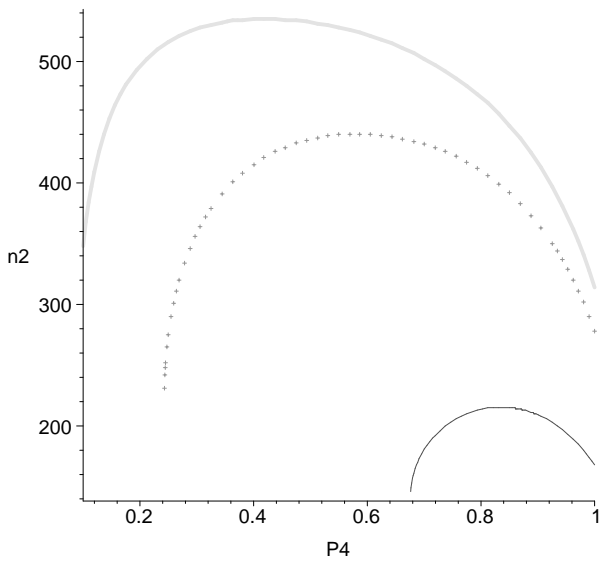

$\mathrm{p} 3=0.8$
$\mathrm{p} 3=0.9$
$\mathrm{p} 3=1$

(a) Effect of fishing on the young class

(b) Effect of fishing on the immature adult class

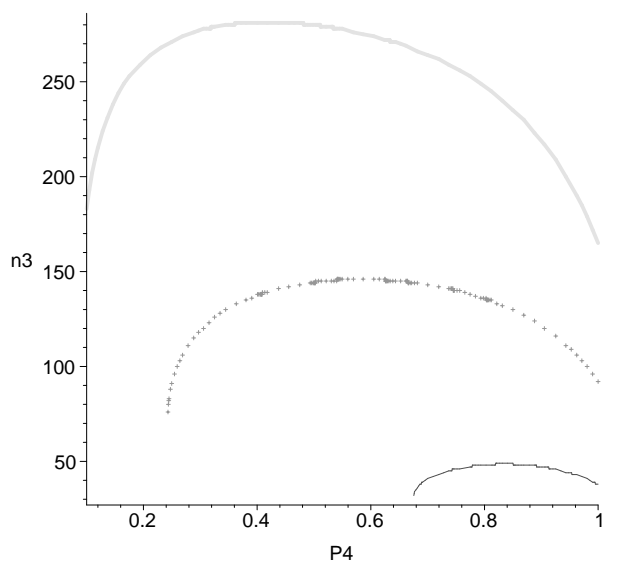

$\mathrm{p} 3=0.8$
$\mathrm{p} 3=0.9$
$\mathrm{p} 3=1$

(c) Effect of fishing on the female class

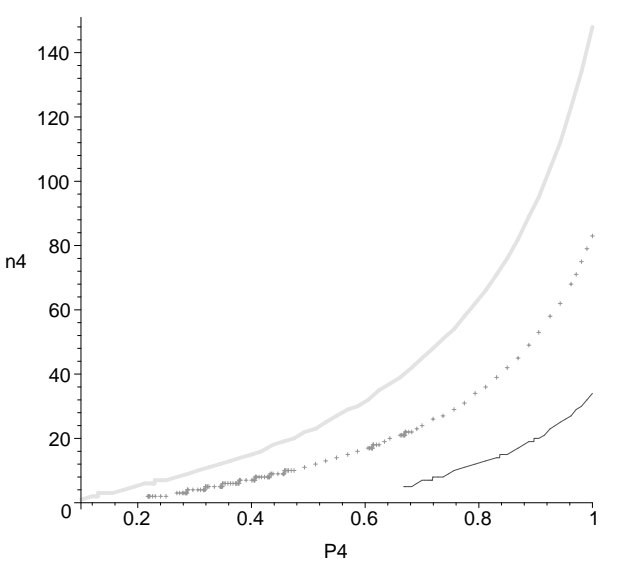

$\mathrm{p} 3=0.8$
$\mathrm{p} 3=0.9$
$\mathrm{p} 3=1$

(d) Effect of fishing on the male class

Figure 6: Effect of fishing on the grouper population for $p_{4} \in[0,1], p_{3} \in\{0.8,0.9,1\}$. 


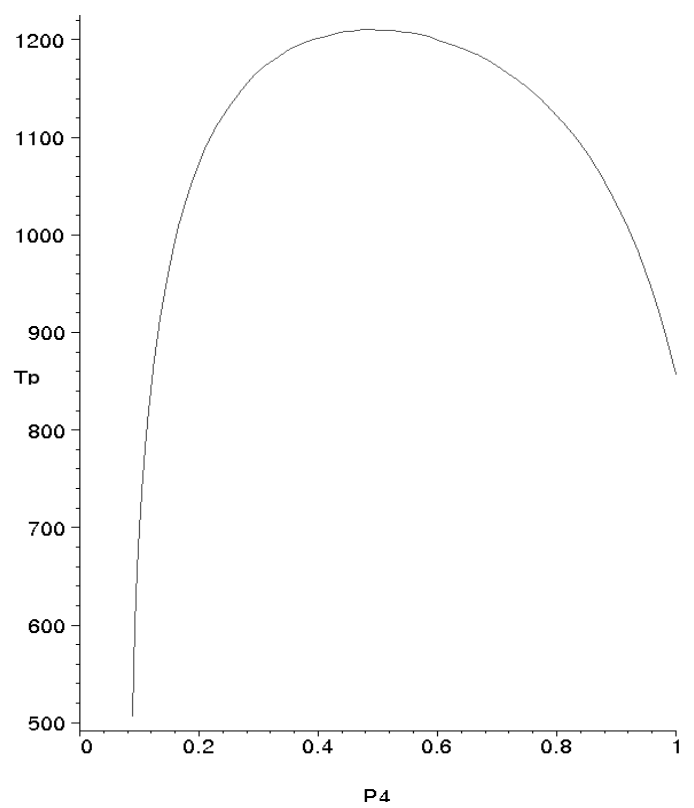

Figure 7: Effect of male fishing on the total population, $T_{p}$ for $p_{3}=1$ and $p_{4} \in[0,1]$.

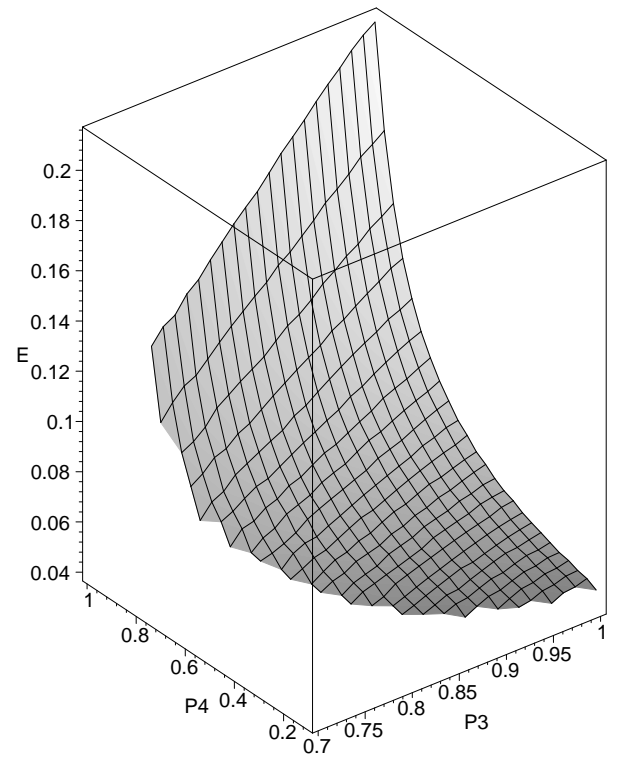

(a) Effect of fishing on the exploitation rate, $E$.

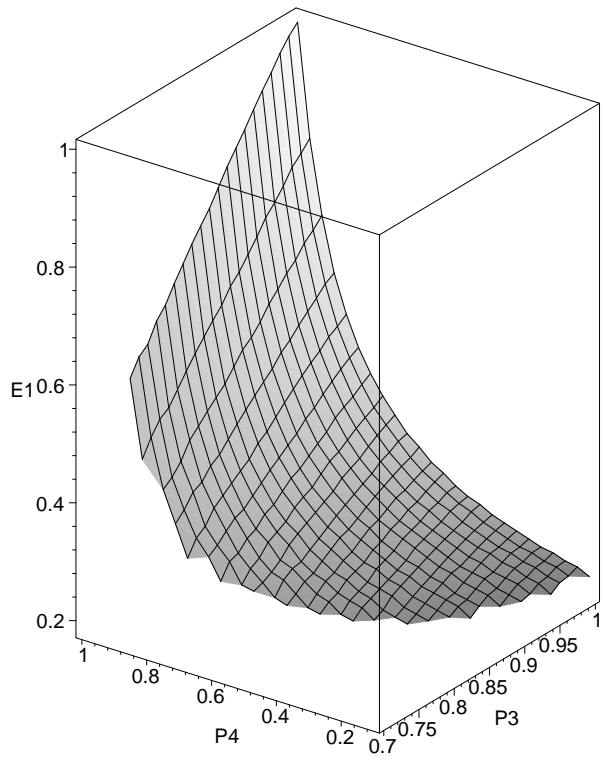

(b) Effect of fishing on $\left(E_{1}=\right.$ $\left.E / E_{\max }\right)$.

Figure 8: Effect of fishing on the exploitation rate for $\left(p_{3}, p_{4}\right) \in[0.7,1] \times[0.1,1]$. 
Mediterranean sea, the number of Epinephelus marginatus decreases from south to north, but with a same population pyramid type. Chauvet suggested that the change in the demographic patterns of these protogynous species is the result of the progressive cooling of the Mediterranean waters from south to north [9] and that the structure of the population pyramid does not depend on fishing, but rather on the survival conditions. Our work comes to support this hypothesis.

One of the most complex characteristics of the grouper is its hermaphroditic nature. Generally in the literature [12], the sexual inversion of Epinephelus marginatus is seen as a spontaneous ectogenic mechanism, i.e. for a given size (or a given age) with a certain individual variability. The optimal age of sexual inversion can be the result of selection and evolution, and thus be an adaptation to the average mating group size over many generations. On the other hand, the observations made by Chauvet [9] plead for a different system, in which the sexual inversion would not be an inevitable process, but would rather depends on social factors, such as the demographic density, the size (or age) group ratios or the sex ratio. So, the sex inversion is fixed neither during evolution nor ontogeny, but remains flexible throughout adult life and can be adjusted to current environmental conditions. In this case, the inversion is jeopardized every year, as well as the succession from female to male classes.

In our case, we assume a fixed size inversion, i.e. the social factors are not taken into account. Therefore, it would be interesting to develop a new model that considers social factors in the sex changing mechanism and to compare both models to experimental or observation data, in order to assess the social impact on the population dynamics.

\section{Acknowledgements}

This work was done with the help of the Morroco-Tunisian project TT/MR 3324. The authors acknowledges the anonymous reviewrs for their careful reading of the manuscript. A.M. acknowledges a Ph.D. grant from the Agence Universitaire de la Francophonie.

\section{References}

[1] W. C. Allee. Animal Aggregations: A Study in General Sociology. University of Chicago Press, 1931.

[2] S. H. Alonzo, M. Mangel. The effects of size-selective fisheries on the stock dynamics of and sperm limitation in sex-changing fish. Fishery Bulletin, 102 (2004), 1-13.

[3] R. J. H. Beverton, S. J. Holt. A review of the lifespans and mortality rates of fish in nature, and their relation to growth and other physiological characteristics. In C. Foundation and G. E. W. Wolstenholme, editors, Ciba foundation colloquia on ageing, 5 (1959), 142-180. Little, Brown. 
[4] J. Bruslé. Synopsis of biological data on the groupers Epinephelus aeneus (Geoffrey Saint Hilaire, 1809) and Epinephelus guaza (Linnaeus, 1758) of the Atlantic Ocean and Mediterranean Sea. FAO Fisheries Synopsis, 129 (1995):1-69.

[5] J. Bruslé, S. Bruslé. Contribution à l'étude de la reproduction de deux espèces de merous (Epinephelus aeneus et Epinephelus guaza) des côtes de Tunisie. Revue des Travaux de l'Institut de Péches Maritimes de Nantes, 39 (1976), No. 3, 313-320.

[6] C. Chauvet. Statut d'Epinephelus guaza (Linnaeus, 1758) et éléments de dynamique des populations méditerranéenne et atlantique. In Boudouresque C.F., M. Avon and V. Gravez, (edit.), Les Espèces Marines Protéger en Méditerranée. GIS Posidonie pub., France, 1991, 255-275.

[7] C. Chauvet. Calcul par otolimetrie de la relation long. T-Age d'Epinephelus guaza (Linnaeus, 1758) de la Côte Nord de la Tunisie. In Rapport de la Commission Internationale Explor. Sci. de la Mer Méditerranée, 27 (1981), 103-106.

[8] C. Chauvet. Etude de la croissance du mérou Epinephelus guaza (Linné, 1758) des côtes tunisiennes. Aquat. Living Resour., 1 (1988), No. 4, 277-288.

[9] C. Chauvet. Relation entre structures démographiques et de frai: Cas du mérou noir Epinephelus marginatus (Lowe, 1834). Observation de longue durée en Méditerrané Occidentale. Private Comm., 2008.

[10] J. M. Cushing. An introduction to structured population dynamics. volume 71 of CBMS-NSF Regional Conference in applied Math. SIAM, Philadelphia, 1998.

[11] F.A.O. La situation mondiale des pêches et de l'aquaculture. (SOFIA). Technical report, FAO, 2002.

[12] M. T. Ghiselin. The evolution of hermaphroditism among animals. Q. Rev. Biol., 44 (1969), No. 2, 189-208.

[13] H. Göthel. Fauna Marina del Mediterráneo. Ediciones Omega, S.A., Barcelona, 1992.

[14] P. C. Heemstra, J. E. Randall. Groupers of the world (family Serranidae, subfamily Epinephelinae). An annotated and illustrated catalogue of the grouper, rockcod, hind, coral grouper and lyretail species known to date. volume 16 of FAO species catalogue. FAO Fish. Synop., Rome, 1993.

[15] A. Laurec, J. C. Le Guen. Dynamique des populations marines exploitées; Tome 1: Concepts et Modèles, volume 45 of Rapports Scientifiques et Techniques. CNEXO, 1981.

[16] M. Liu, Y. Sadovy. The influence of social factors on adult sex change and juvenile sexual differentiation in a diandric, protogynous epinepheline, Cephalopholis boenak (Pisces, Serranidae). J. Zool (London), 264 (2004), No. 3, 239-248. 
[17] T. J. Quinn, R. B. Deriso. Quantitative fish dynamics. Oxford University Press, New york USA, 1999.

[18] J. E. Randall. Coastal Fishes of Oman. University of Hawaii Press, Honolulu, 1995.

[19] R. R. Warner. Sex change and the size-advantage model. Trends Ecol. Evol., 3(1988) No. 6, 133-136.

[20] M. Zabala, A. Garcia-Rubies, P. Louisy, E. Sala. Spawning behaviour of the Mediterranean dusky grouper Epinephelus marginatus (Lowe, 1834) (Pisces, Serranidae) in the Medes Islands Marine Reserve (NW Mediterranean, Spain). Scientia Marina, 61(1997), No. 1, 65-77.

[21] M. Zabala, P. Louisy, A. Garcia-Rubies, V. Gracia. Socio-behavioural context of reproduction in the Mediterranean dusky grouper Epinephelus marginatus (Lowe, 1834) (Pisces, Serranidae) in the Medes Islands Marine Reserve (NW Mediterranean, Spain). Scientia Marina, 61 (1997), No. 1,79-98. 